\title{
A geometric proof of a theorem on antiregularity of generalized quadrangles
}

\author{
Anna Y. Pun · Philip P. W. Wong
}

Received: 17 January 2011 / Revised: 17 June 2011 / Accepted: 19 September 2011 / Published online: 16 October 2011

(C) The Author(s) 2011. This article is published with open access at Springerlink.com

\begin{abstract}
A geometric proof is given in terms of Laguerre geometry of the theorem of Bagchi, Brouwer and Wilbrink, which states that if a generalized quadrangle of order $s>1$ has an antiregular point then all of its points are antiregular.
\end{abstract}

Keywords Antiregularity · Generalized quadrangles · Codes · Laguerre geometry

Mathematics Subject Classification (2000) $\quad 51 \mathrm{~B} 15 \cdot 51 \mathrm{E} 12 \cdot 51 \mathrm{E} 22$

\section{Introduction}

In design theory, in particular the study of incidence structures, characterizations of the classical examples play a basic role in the development of classification results as well as the linkage of various incidence geometries. In the case of generalized quadrangles, some of these results provide short and elegant proofs of classical theorems in circle geometries [9].

In the special case of a generalized quadrangle of order $s$, one of the oldest combinatorial characterization results is that of $\mathcal{W}(s)$, namely, that a generalized quadrangle of order $s>1$ is isomorphic to $\mathcal{W}(s)$ if and only if all of its points are regular [2]. Recall that $\mathcal{W}(s)$ consists of the points of $P G(3, s)$ and the totally isotropic lines with respect to a symplectic polarity (see for example [4,5]). As is well-known, the dual of $\mathcal{W}(s)$ is $\mathcal{Q}(4, s)$, the points and lines of the parabolic quadric in $P G(4, s)$. Furthermore, $\mathcal{Q}(4, s)$ is self dual if and only if $s$ is even.

Communicated by J. D. Key.

This work was partially supported by a grant from the Research Grants Council of the HKSAR, China (Project number: HKU7060/11P).

A. Y. Pun · P. P. W. Wong $(\bowtie)$

Department of Mathematics, The University of Hong Kong, Pokfulam Road, Hong Kong, China e-mail: ppwwong@maths.hku.hk

A. Y. Pun

e-mail: annapunying@gmail.com 
When $s$ is odd, all points of $\mathcal{Q}(4, s)$ are antiregular. Whether the converse is true, namely, a generalized quadrangle of (odd) order $s>1$ is isomorphic to $\mathcal{Q}(4, s)$ if all of its points are antiregular, is an open problem (see for example [10, Problem A.2.4]).

In this direction, there is the following characterization result by Mazzocca [6] and Payne and Thas [7]: Let $\mathcal{S}$ be a generalized quadrangle of order $s>1$, having an antiregular point $x$. Then $\mathcal{S}$ is isomorphic to $\mathcal{Q}(4, s)$ if and only if there is a point $y$ collinear with $x, y \neq x$, for which the associated affine plane is Desarguesian. In [7] the relation between antiregularity of a generalized quadrangle and Laguerre geometry is given a thorough treatment and the linkage to the classical example is thus provided by the classical Laguerre plane.

In view of the above result it is of interest to study generalized quadrangles with an antiregular point. Remarkably, in their study on the dimensions of the binary codes of certain generalized quadrangles, Bagchi et al. [1] have proven that for such a generalized quadrangle all of its points are antiregular [1, Theorem 3.8]. Since the proof of the result is in terms of codes, the question has been raised as to whether a geometric proof can be given [10, Problem A.2.9]. Accordingly, we recall the basic results we need from the theory of generalized quadrangles in Sect. 2, review the relevant results in [1] and provide elementary proofs for non-experts in Sect. 3, recall the results of [7] and study further the circle geometry of Laguerre in some details in Sect. 4, and give a proof of the theorem of Bagchi, Brouwer and Wilbrink cited above in terms of the properties of these circles in Sect. 5.

\section{Generalized quadrangles and antiregularity}

In this section, we study the concept of antiregularity in a generalized quadrangle. First we recall the definitions [8].

Definition 2.1 A finite generalized quadrangle (GQ) of order $(s, t), s, t \in \mathbb{N}$, is an incidence structure $\mathcal{S}=(\mathcal{P}, \mathcal{B}, \mathbf{I})$ in which $\mathcal{P}$ and $\mathcal{B}$ are nonempty sets of objects called points and lines, respectively, and for which $\mathbf{I}$ is a symmetric point-line incidence relation satisfying the following axioms:

$G Q 1$ Each point is incident with $1+t$ lines and two distinct points are incident with at most one line.

$G Q 2$ Each line is incident with $1+s$ points and two distinct lines are incident with at most one point.

$G Q 3$ If $y$ is a point and $L$ is a line not incident with $y$, then there is a unique pair $(z, M) \in$ $\mathcal{P} \times \mathcal{B}$ for which $y \mathbf{I} M \mathbf{I} z \mathbf{I} L$.

The GQ $\mathcal{S}$ is said to have $\operatorname{order}(s, t)$; if $s=t, \mathcal{S}$ is said to have order $s$. There is a natural point-line duality for GQ and we assume without further notice the dual theorems and definitions.

Given a GQ $\mathcal{S}=(\mathcal{P}, \mathcal{B}, \mathbf{I})$, consider the graph $\Gamma=(\mathcal{P}, E)$, where $\{y, z\} \in E(y \neq z$ and $y, z \in \mathcal{P}$ ) if and only if there exists a line $L$ in $\mathcal{B}$ such that both $y$ and $z$ are incident with $L$. Write $y \sim z$ and say $y$ and $z$ are connected (or $y$ is connected to $z$ ) if $\{y, z\} \in E$, and $y \nsim z$ if $y$ and $z$ are not connected (in which case we also refer to $y$ and $z$ as non-connected points). For $y \in \mathcal{P}$, let $y^{\perp}=\{y\} \cup\{z \in \mathcal{P} \mid z \sim y\}$.

Definition 2.2 Given a GQ $\mathcal{S}=(\mathcal{P}, \mathcal{B}, \mathbf{I})$, two distinct points $y$, $z$ form an antiregular pair if $y \nsim z$ and $\left|x^{\perp} \cap y^{\perp} \cap z^{\perp}\right| \leq 2$ for all $x \in \mathcal{P} \backslash\{y, z\}$. A point $z$ is antiregular if $y$ and $z$ form an antiregular pair for all $y \in \mathcal{P} \backslash z^{\perp}$. 
Let $\mathcal{S}=(\mathcal{P}, \mathcal{B}, \mathbf{I})$ be a GQ of order $s, s \geq 3$. We recall the following facts [8].

Lemma 2.3 The points $y, z$ form an antiregular pair if and only if $y \nsim z$ and $\left|x^{\perp} \cap y^{\perp} \cap z^{\perp}\right|=$ 0 or 2 for all $x \in \mathcal{P} \backslash\left(y^{\perp} \cup z^{\perp}\right)$.

Lemma 2.4 If the points $y$ and $z$ form an antiregular pair then $s$ is odd.

Definition 2.5 The set $y^{\perp} \cap z^{\perp}$ for a pair of distinct points $(y, z)$ is called the trace of $(y, z)$ and is denoted by $\operatorname{tr}(y, z)$.

We now describe another characterization of antiregularity using linear algebra [1]. Denote by $\mathbb{F}_{2}\{\mathcal{P}\}$ the vector space over the finite field $\mathbb{F}_{2}$ with basis $\{\{x\} \mid x \in \mathcal{P}\}$. There is a canonical injection

$$
\iota: 2^{\mathcal{P}} \rightarrow \mathbb{F}_{2}\{\mathcal{P}\}
$$

given by

$$
S \mapsto \sum_{x \in S}\{x\} .
$$

We shall identify $S \subset \mathcal{P}$ with $\iota(S) \in \mathbb{F}_{2}\{\mathcal{P}\}$. Let $\pi$ be the $\mathbb{F}_{2}$-linear map from $\mathbb{F}_{2}\{\mathcal{P}\}$ to itself given by $\pi(\{x\})=x^{\perp} \backslash\{x\}$ for any $x \in \mathcal{P}$. We write $\pi(A)$ as $\pi_{A}$ for all $A \subset \mathcal{P}$ and $\pi(\{x\})$ as $\pi_{x}$ for all $x \in \mathcal{P}$.

Lemma 2.6 For all $A, B \subset \mathcal{P}, \pi_{A}+\pi_{B}=\pi_{A+B}$.

Proof This follows from the fact that $A+B=(A \cup B) \backslash(A \cap B)$.

Lemma 2.6 will be used freely below and no explicit mention will be made of it. Using the map $\pi$ we describe another characterization of an antiregular pair of points in a GQ of odd order $s$.

Theorem 2.7 [1] Let $\mathcal{S}=(\mathcal{P}, \mathcal{B}, \mathbf{I})$ be a $G Q$ of odd order $s$. Two non-connected points $y, z$ of $\mathcal{S}$ form an antiregular pair if and only if $\pi_{y}+\pi_{z}=\pi_{\operatorname{tr}(y, z)}$.

Proof Suppose $y, z$ form an antiregular pair. By Lemma 2.3, for every point $x \in \mathcal{P} \backslash\left(y^{\perp} \cup\right.$ $\left.z^{\perp}\right),\left|x^{\perp} \cap y^{\perp} \cap z^{\perp}\right|=0$ or 2 . Hence every such point $x$ is connected to an even number of points in $\operatorname{tr}(y, z)$. Since $s$ is odd, this is also true for the points $y$ and $z$. It follows that $\pi_{y}+\pi_{z}=\pi_{\operatorname{tr}(y, z)}$.

Conversely, suppose $\pi_{y}+\pi_{z}=\pi_{\operatorname{tr}(y, z)}$. Then for every point $x \in \mathcal{P} \backslash\left(y^{\perp} \cup z^{\perp}\right), \mid x^{\perp} \cap$ $y^{\perp} \cap z^{\perp} \mid$ is even. In view of Lemma 2.3, we need only show that $\left|x^{\perp} \cap y^{\perp} \cap z^{\perp}\right|=0$ or 2 for every such $x$. To this end, define $A_{i}=\left\{x \in \mathcal{P} \backslash\left(y^{\perp} \cup z^{\perp}\right)|| x^{\perp} \cap y^{\perp} \cap z^{\perp} \mid=i\right\}$. By the usual counting arguments, we have the equalities:

$$
\begin{aligned}
& \sum_{i=0}^{s+1} i\left|A_{i}\right|=\left|\left\{(u, v) \mid u \in \mathcal{P} \backslash\left(y^{\perp} \cup z^{\perp}\right), v \in \operatorname{tr}(y, z), u \sim v\right\}\right|=(s+1-2) s(s+1)=s^{3}-s, \\
& \quad \sum_{i=0}^{s+1} i(i-1)\left|A_{i}\right| \\
& \quad=\left|\left\{\left(u, v_{i}, v_{j}\right) \mid u \in \mathcal{P} \backslash\left(y^{\perp} \cup z^{\perp}\right), v_{i}, v_{j} \in \operatorname{tr}(y, z), v_{i} \neq v_{j}, u \sim v_{i}, u \sim v_{j}\right\}\right| \\
& =(s+1) s(s+1-2) \\
& =s^{3}-s .
\end{aligned}
$$


Subtracting we obtain $\sum_{i=0}^{s+1} i(i-2)\left|A_{i}\right|=0$. Since $\left|A_{i}\right|=0$ for all odd $i$, this gives

$$
0=\sum_{4 \leq i \leq s+1, i \text { even }} i(i-2)\left|A_{i}\right|,
$$

which implies that $\left|A_{i}\right|=0$ for all $i \geq 3$, as we wished.

We shall see in the next section that the following fact has interesting consequences [1]. We give here an elementary proof for non-experts.

Theorem 2.8 [1] Let $\mathcal{S}=(\mathcal{P}, \mathcal{B}, \mathbf{I})$ be a $G Q$ of odd order $s$, and $z \in \mathcal{P}$. The following conditions are equivalent:

(i) For every $y \in \mathcal{P} \backslash z^{\perp}, \pi_{y}+\pi_{z}=\pi_{y^{\perp} \cap z^{\perp}}$.

(ii) For every $y \in \mathcal{P} \backslash z^{\perp}$, there exists $Y \subset z^{\perp}$ such that $\pi_{y}=\pi_{Y}$.

Proof (i) $\Rightarrow$ (ii) Take $Y=\{z\} \cup\left(y^{\perp} \cap z^{\perp}\right)$.

(ii) $\Rightarrow$ (i) Write $y^{\perp} \cap z^{\perp}=\left\{u_{1}, u_{2}, \ldots, u_{s+1}\right\}$ and let $\mathcal{L}_{z}=\left\{L_{1}, L_{2}, \ldots, L_{s+1}\right\}$ be the pencil of lines on $z$ where $u_{i}$ is incident with $L_{i}$ for $1 \leq i \leq s+1$. We study the intersection of Y with each line $L_{i}$. We first show that either $L_{i} \backslash\left\{u_{i}, z\right\} \subset Y$ or $\left(L_{i} \backslash\left\{u_{i}, z\right\}\right) \cap Y=\emptyset$. It suffices to show that there do not exist two points $p, q$ such that $p \in\left(L_{i} \backslash\left\{u_{i}, z\right\}\right) \backslash Y$ and $q \in\left(L_{i} \backslash\left\{u_{i}, z\right\}\right) \cap Y$. Suppose the contrary. Then $p$ is connected to $\left|L_{i} \cap Y\right|$ points in $Y$ while $q$ is connected to $\left|L_{i} \cap Y\right|-1$ points in $Y$. Since $\pi_{y}=\pi_{Y}$, every point not in $y^{\perp} \backslash\{y\}$ connects to an even number of points in $Y$. In particular, both $p$ and $q$ connect to an even number of points in $Y$. This implies that both $\left|L_{i} \cap Y\right|$ and $\left|L_{i} \cap Y\right|-1$ are even, which is a contradiction.

Next we deduce that there are two possibilities for the intersection of $Y$ with each line $L_{i}$, namely, $L_{i} \cap Y=L_{i} \backslash\left\{u_{i}\right\}$ or $L_{i} \cap Y=\left\{u_{i}, z\right\}$. In the first case we call $L_{i}$ a line of type (I), and in the second case we call it a line of type (II). If $L_{i} \backslash\left\{u_{i}, z\right\} \subset Y$, then $L_{i}$ is of type (I). Indeed, since $\pi_{y}=\pi_{Y}$, every point in $y^{\perp} \backslash\{y\}$ connects to an odd number of points in $Y$. In particular, $u_{i}$ is connected to an odd number of points in $Y$. This means that, as $\left|\left(L_{i} \backslash\left\{u_{i}, z\right\}\right) \cap Y\right|=s-1$, which is even, $z$ must be in $Y$. Now if $u_{i}$ is also in $Y$, then for $p \in L_{i} \backslash\left\{u_{i}, z\right\}, p$ is connected to an odd number of points in $Y$. This is a contradiction since $p \notin y^{\perp} \backslash\{y\}$ and we have noted in the paragraph above that in this case the number must be even. Similar arguments show that if $\left(L_{i} \backslash\left\{u_{i}, z\right\}\right) \cap Y=\emptyset$, then $L_{i}$ is a line of type (II).

Finally we show that we can change pairs of lines of type (I) into type (II) without changing $\pi_{Y}$. Note that $\left|\left\{u_{1}, u_{2}, \ldots, u_{s+1}\right\} \cap Y\right|$ is even as $y \notin y^{\perp} \backslash\{y\}$, i.e. there is an even number of lines of type (II) in $\mathcal{L}_{z}$. Since $s+1$ is even, the number of lines of type (I) is also even, and so we may assume that all lines are of type (II), i.e. $Y=\left\{z, u_{1}, u_{2}, \ldots, u_{s+1}\right\}$. Thus $\pi_{Y}=\pi_{z}+\pi_{y^{\perp} \cap z^{\perp}}$, which gives (i).

So suppose $u_{i}, u_{j}$ are two distinct points not in $Y$, i.e. $L_{i}, L_{j}$ are of type (I). We show that if we convert them into lines of type (II), then $\pi_{Y^{*}}=\pi_{Y}$, where $Y^{*}=\left(Y \backslash\left(L_{i} \cup L_{j}\right)\right) \cup$ $\left\{u_{i}, u_{j}, z\right\}$. To this end, let $q \in \mathcal{P}$ and let $n$ be the number of points in $Y$ connected to $q$. We first consider the case where $q \notin\left(L_{i} \cup L_{j}\right)$. There are three possibilities: $(a) q \sim u_{i}$ and $q \sim u_{j}$. Here $q$ is connected to $n+2$ points in $Y^{*} ;(b) q$ is connected to exactly one point in $\left\{u_{i}, u_{j}\right\}$. Here $q$ is connected to $n+1-1=n$ points in $Y^{*} ;(c) q \nsim u_{i}$ and $q \nsim u_{j}$. Here $q$ is connected to $n-2$ points in $Y^{*}$. Next we consider the case where $q \in\left(L_{i} \cup L_{j}\right)$. Again there are three possibilities: (a) $q=z$. Here $q$ is connected to $n-2(s-1)+2$ points in $Y^{*}$; (b) $q=u_{i}$ or $q=u_{j}$. Here $q$ is connected to $n-(s-1)$ points in $Y^{*}$; (c) $q \in\left(L_{i} \cup L_{j}\right) \backslash\left\{u_{i}, u_{j}, z\right\}$. Here $q$ is connected to $n-(s-2)+1=n-(s-3)$ points in $Y^{*}$. 
Since $s$ is odd, the parity of $n$ is invariant under the change in types of $L_{i}, L_{j}$. As a result, $\pi_{Y^{*}}=\pi_{Y}$. This completes the proof of the theorem.

\section{Antiregularity and dimensions of GQ-related binary codes}

In this section we recall the binary codes spanned by the point neighbourhoods of a generalized quadrangle $\mathcal{S}=(\mathcal{P}, \mathcal{B}, \mathbf{I})$ of odd order $s$ [1]. We shall study their dimensions and the geometrical implications they have on the incidence structure itself. The results are all contained in [1] but again we shall provide elementary proofs for non-experts. Recall the vector space $\mathbb{F}_{2}\{\mathcal{P}\}$ of Sect. 2 . This vector space is provided with the standard symmetric bilinear form (,) defined by $(\{x\},\{x\})=1$ and $(\{x\},\{y\})=0$ for $x, y \in \mathcal{P}, x \neq y$. For any $U \subset \mathbb{F}_{2}\{\mathcal{P}\}$, define $U^{\perp}=\left\{v \in \mathbb{F}_{2}\{\mathcal{P}\} \mid(u, v)=0 \forall u \in U\right\}$.

Consider the code $P=\pi\left(\mathbb{F}_{2}\{\mathcal{P}\}\right)=\left\langle\pi_{x} \mid x \in \mathcal{P}\right\rangle_{\mathbb{F}_{2}}$, and for $z \in \mathcal{P}$ the subcode $P_{z}=$ $\left\langle\pi_{x} \mid x \in z^{\perp}\right\rangle_{\mathbb{F}_{2}}$. We first note the following upper bound for the dimension of $P$.

Lemma 3.1 $\operatorname{dim} P \leq(s+1)\left(s^{2}+1\right) / 2$.

Proof It is readily verified that $P$ is a subspace of $P^{\perp}$. Then $\operatorname{dim} P \leq \operatorname{dim} P^{\perp}$ and so $2 \operatorname{dim} P \leq \operatorname{dim} P^{\perp}+\operatorname{dim} P=\operatorname{dim}\langle\{x\} \mid x \in \mathcal{P}\rangle_{\mathbb{F}_{2}}=|\mathcal{P}|=(s+1)\left(s^{2}+1\right)$.

Clearly, $\operatorname{dim} P_{z}$ is a lower bound for $\operatorname{dim} P$.

Theorem $3.2 \operatorname{dim} P_{z}=s^{2}+1$.

Proof By Lemma 2.6, $\left\{A \subseteq z^{\perp} \mid \pi_{A}=0\right\}$ is a vector space. Since $\operatorname{dim} P_{z}=\left|z^{\perp}\right|-\operatorname{dim}\{A \subseteq$ $\left.z^{\perp} \mid \pi_{A}=0\right\}$, we study the structure of $A \subseteq z^{\perp}$ which satisfies $\pi_{A}=0$.

Denote by $\mathcal{L}_{z}$ the set of all lines incident with $z$, and for $L \in \mathcal{L}_{z}$, write $L^{\prime}=L \backslash\{z\}$. Let $A \subseteq z^{\perp}$ such that $\pi_{A}=0$. We claim that

$$
A=\bigcup_{L \in \mathcal{T}} L^{\prime}
$$

for some $\mathcal{T} \subseteq \mathcal{L}_{z}$ with $|\mathcal{T}|$ even. To see this we first note that $\pi_{A}=0$ if and only if every point is connected to an even number of points in $A$. Now consider $A \cap L^{\prime}$ for $L \in \mathcal{L}_{z}$. If there exist $p, q \in L^{\prime}$ such that $p \in A$ but $q \notin A$, then, since every point in $z^{\perp} \backslash L$ is collinear with neither $p$ nor $q, p$ is connected to $|A \cap L|-1$ points in $A$ and $q$ is connected to $|A \cap L|$ points in $A$. However, $|A \cap L|-1$ and $|A \cap L|$ cannot be both even and we have a contradiction. Hence $A \cap L^{\prime}=\emptyset$ or $L^{\prime}$, i.e. either $L^{\prime} \subseteq A$ or $L^{\prime} \cap A=\emptyset$. Next we check that $z \notin A$. Indeed, if $z \in A$, then for $L \in \mathcal{L}_{z}$, every point in $L^{\prime}$ is connected to either 1 or $s$ points in $A$ which is a contradiction as $s$ is odd. Finally, since $\left|\left(z^{\perp} \backslash\{z\}\right) \cap A\right|$ is even and $s$ is odd, $|\mathcal{T}|$ is even. This proves the claim.

Conversely we claim that by taking any nonempty subset $\mathcal{T} \subseteq \mathcal{L}_{z}$ with $|\mathcal{T}|$ even, $\pi_{A}=0$, where $A=\bigcup_{L \in \mathcal{T}} L^{\prime}$. We verify this by checking that every point is connected to an even number of points of $A$. If $x \in \mathcal{P} \backslash z^{\perp}$, then $x$ is incident with exactly one point in every $L$ in $\mathcal{T}$ and $x \nsim z$. This implies that $x$ is connected to an even number of points in $A$. If $y \in z^{\perp} \backslash A$, then $y$ is not connected to any point in $A$ if $y$ is different from $z$. Note that $z$ is connected to all points of $A$, a set of even cardinality. Finally, if $w \in A$ let $L_{w}$ be the line in $\mathcal{T}$ containing $w$. Then $w$ is connected to all points in $\left(L_{w} \cap A\right) \backslash\{w\}$, i.e. $s-1$ points in $A$. As $s$ is odd, $w$ is connected to an even number of points in $A$. This proves the claim.

In conclusion, $\operatorname{dim}\left\{A \subseteq z^{\perp} \mid \pi_{A}=0\right\}=\left|\mathcal{L}_{z}\right|-1=s$. Thus $\operatorname{dim} P_{z}=\left|z^{\perp}\right|-\operatorname{dim}\{A \subseteq$ $\left.z^{\perp} \mid \pi_{A}=0\right\}=s\left|\mathcal{L}_{z}\right|+1-s=s(s+1)+1-s=s^{2}+1$. 
The computation of the dimension of these codes is related to the geometry of the GQ in the following way. Let $z$ be a point of a GQ of odd order $s$. We are interested in determining whether it is an antiregular point. By Theorems 2.7 and 2.8, this is equivalent to asking whether it is true that for every $y \in \mathcal{P} \backslash z^{\perp}$, there exists $Y \subset z^{\perp}$ such that $\pi_{y}=\pi_{Y}$. Now the last statement is equivalent to having $P=P_{z}$, which in turn is equivalent to having $\operatorname{dim} P$ $=\operatorname{dim} P_{z}$. It follows that if $z$ is an antiregular point of $\mathcal{S}$, then $\operatorname{dim} P=s^{2}+1$, by Theorem 3.2, and, by the same theorem, this implies that for any other point $z^{\prime}, \operatorname{dim} P=\operatorname{dim} P_{z^{\prime}}$, i.e. $P=P_{z^{\prime}}$. As explained above, this is equivalent to saying that $z^{\prime}$ is antiregular. We have recounted the following theorem of Bagchi, Brouwer and Wilbrink:

Theorem 3.3 [1] If a finite generalized quadrangle of order s has one antiregular point then all of its points are antiregular.

Proof In the above the result is obtained under the assumption that the order of the GQ is odd. But this assumption is a consequence of the hypothesis, by Lemma 2.4.

\section{Laguerre geometry}

We begin by summarizing some of the basic facts in Laguerre geometry and then recall the canonical correspondence between a finite Laguerre plane of odd order and a generalized quadrangle with an antiregular point [7].

Definition 4.1 A finite Laguerre plane is a finite incidence structure $\mathcal{L}=\left(\mathcal{P}, \mathcal{B}_{1} \cup \mathcal{B}_{2}\right.$, I $)$ with $\mathcal{P}$ the point set, $\mathcal{B}_{1}$ the line set, $\mathcal{B}_{2}$ the circle set, and $\mathbf{I}$ the incidence relation satisfying the following axioms:

$L 1$ Each point is incident with exactly one element of $\mathcal{B}_{1}$.

L2 Each line and each circle intersect in exactly one point.

L3 Any three pairwise non-collinear points are incident with exactly one circle.

$L 4$ If $x$ and $y$ are non-collinear points and $C$ is a circle incident with $x$ but not $y$, then there is a unique circle $C^{\prime}$ incident with $y$ and intersecting $C$ only at $x$.

L5 There is a point $x$ and a circle $C$ with $x$ not incident with $C$; each circle is incident with at least three points.

Let $\mathcal{L}=\left(\mathcal{P}, \mathcal{B}_{1} \cup \mathcal{B}_{2}, \mathbf{I}\right)$ be a Laguerre plane and $x \in \mathcal{P}$. The internal structure $\mathcal{L}_{x}=$ $\left(\mathcal{P}_{x}, \mathcal{B}_{x}, \mathbf{I}_{x}\right)$ is defined as follows: $\mathcal{P}_{x}$ consists of all points of $\mathcal{P}$ not collinear with $x, \mathcal{B}_{x}$ consists of all lines not incident with $x$ and all circles incident with $x$, and $\mathbf{I}_{x}=\mathbf{I} \cap\left(\mathcal{P}_{x} \times \mathcal{B}_{x}\right)$. It is readily verified that $\mathcal{L}_{x}$ is an affine plane, and the order of the affine plane is independent of the choice of $x$. This order is the order of the Laguerre plane $\mathcal{L}$. For a Laguerre plane of order $s$, we have that $|\mathcal{P}|=s(s+1),\left|\mathcal{B}_{1}\right|=s+1,\left|\mathcal{B}_{2}\right|=s^{3}$, each line is incident with $s$ points, each circle is incident with $s+1$ points, and each point is incident with 1 line and $s^{2}$ circles.

We next recall the canonical correspondence between finite Laguerre planes of odd order and the generalized quadrangles with a distinguished antiregular point [7]. Given a Laguerre plane $\mathcal{L}=\left(\mathcal{P}, \mathcal{B}_{1} \cup \mathcal{B}_{2}\right.$, I $)$ of odd order $s$, consider the following incidence structure $\mathcal{S}=\left(\mathcal{P}^{*}, \mathcal{B}^{*}, \mathbf{I}^{*}\right)$, where the point set $\mathcal{P}^{*}$ consists of (i) the symbol ( $\left.\infty\right)$, (ii) the points of $\mathcal{P}$, and (iii) the circles in $\mathcal{B}_{2}$; and the line set $\mathcal{B}^{*}$ consists of (a) the lines in $\mathcal{B}_{1}$ with $(\infty)$ added to each line, and (b) the pencils of mutually tangent circles of $\mathcal{L}$ with the point of tangency added to each pencil. A point $x$ of type (ii) is on a line of type (a) and on each pencil of mutually tangent circles at $x$. A point $C$ of type (iii) is on each pencil in (b) containing $C$. Then it can be shown that $\mathcal{S}$ is a generalized quadrangle of order $s$ and $(\infty)$ is an antiregular point. 
Conversely, let $\mathcal{S}=\left(\mathcal{P}^{*}, \mathcal{B}^{*}, \mathbf{I}^{*}\right)$ be a generalized quadrangle of order $s$ with a fixed antiregular point $x_{\infty}$. By Lemma 2.4, $s$ is odd. Let $\mathcal{L}=\left(\mathcal{P}, \mathcal{B}_{1} \cup \mathcal{B}_{2}\right.$, I $)$, where the point set $\mathcal{P}=x_{\infty}^{\perp} \backslash\left\{x_{\infty}\right\}$, the line set $\mathcal{B}_{1}$ consists of the lines in $\mathcal{B}^{*}$ incident with $x_{\infty}$ but with $x_{\infty}$ deleted from each line, the circle set $\mathcal{B}_{2}$ consists of the sets $\operatorname{tr}\left(y, x_{\infty}\right)$ where $y \in \mathcal{P}^{*} \backslash x_{\infty}^{\perp}$, and $\mathbf{I}$ is the natural incidence. Then it can be shown that $\mathcal{L}$ is a Laguerre plane of order s. We summarize the discussion above in the following lemma:

Lemma 4.2 [7] The following concepts are equivalent:

(i) A Laguerre plane of odd order s.

(ii) A generalized quadrangle of order s with an antiregular point $x$.

It is also readily seen that the correspondence between the two structures is canonical in the sense that if we apply the corresponding procedures consecutively we obtain a structure isomorphic to the one we started with.

We now prepare some lemmas to be used in the next section where a geometric proof of Theorem 3.3 is given.

Lemma 4.3 Let $\mathcal{L}=\left(\mathcal{P}, \mathcal{B}_{1} \cup \mathcal{B}_{2}, \mathbf{I}\right)$ be a Laguerre plane of odd order $\mathrm{s} \geq 3$. Let $C, C^{\prime} \in \mathcal{B}_{2}$ be two circles tangent at $x \in \mathcal{P}$. Let $y, z \in \mathcal{P} \backslash\{x\}$ be two distinct points on $C$. Then there exists a unique circle $C^{\prime \prime} \in \mathcal{B}_{2} \backslash\{C\}$ such that $y, z$ are on $C^{\prime \prime}$ and $C^{\prime \prime}$ and $C^{\prime}$ are tangent.

The above lemma, formulated in terms of the generalized quadrangle associated with the Laguerre plane, is Lemma 2.2.1 of [3]. Hence there is no need to repeat the proof.

Lemma 4.4 Let $\mathcal{L}=\left(\mathcal{P}, \mathcal{B}_{1} \cup \mathcal{B}_{2}\right.$, I) be a Laguerre plane of odd order $s \geq 3$. Let $C \in \mathcal{B}_{2}$ be a circle with points $x_{0}, x_{1}, \ldots, x_{s}$ on $C$. Let a be a point not on $C$ and collinear with $x_{0}$. Let $C_{t}\left(a, x_{i}\right)$ be the circle on $a$ and tangent to $C$ at $x_{i}, 1 \leq i \leq s$. Then: $(i)$ For every $b \in \mathcal{P} \backslash\left\{x_{0}, \ldots, x_{s}, a\right\}, b$ is on either 0 or 2 circles in $\left\{C_{t}\left(a, x_{i}\right) \mid 1 \leq i \leq s\right\}$. (ii) $C_{t}\left(a, x_{i}\right)$ and $C_{t}\left(a, x_{j}\right)$ have exactly 2 points in common for all $i, j$, where $1 \leq i<j \leq s$.

Proof (i) Let $b$ be a point on $C_{t}\left(a, x_{i}\right)$ for some $i \in\{1, \ldots, s\}$ and $b \neq a$. Then by Lemma 4.3, there exists a unique circle $C_{t}\left(a, x_{j}\right)$ on $b$ for some $j \neq i$. (ii) This follows from (i). (Alternatively, observe that if $C_{t}\left(a, x_{i}\right)$ and $C_{t}\left(a, x_{j}\right)$ are tangent at $a$, then $C_{t}\left(a, x_{i}\right), C_{t}\left(a, x_{j}\right)$ and $C$ are three pairwise tangent circles with three points of tangency $x_{i}, x_{j}$ and $a$. In the corresponding GQ provided by Lemma 4.2 we then have a triangle, which is impossible.)

The above lemma, formulated in terms of the generalized quadrangle associated with the Laguerre plane, is also immediate from Lemma 2.2.1 cited above.

Lemma 4.5 Let $\mathcal{L}=\left(\mathcal{P}, \mathcal{B}_{1} \cup \mathcal{B}_{2}\right.$, I) be a Laguerre plane of odd order $s \geq 3$. Let $C, C^{\prime} \in \mathcal{B}_{2}$ be two distinct circles which are not tangent to each other. Then:

(i) If $C$ and $C^{\prime}$ have no common point, then for any point a which is on neither $C$ nor $C^{\prime}$, there is an even number of circles containing a and tangent to both $C$ and $C^{\prime}$.

(ii) If $C$ and $C^{\prime}$ have two common points, say $x_{0}$ and $x_{1}$, then

(a) for each a which is on neither $C$ nor $C^{\prime}$ and collinear with $x_{i}, i=0$ or 1 , there is an odd number of circles containing $a$ and tangent to both $C$ and $C^{\prime}$;

(b) for each a which is on neither $C$ nor $C^{\prime}$ and collinear with neither $x_{0}$ nor $x_{1}$, there is an even number of circles containing $a$ and tangent to both $C$ and $C^{\prime}$. 
Proof (i) Let $\left\{x_{0}, x_{1}, \ldots, x_{s}\right\}$ and $\left\{y_{0}, y_{1}, \ldots, y_{s}\right\}$ be the sets of points on $C$ and $C^{\prime}$ respectively, where $x_{0}$ and $y_{0}$ are collinear with $a$. Let $\mathcal{C}=\left\{C_{t}\left(a, x_{i}\right) \mid 1 \leq i \leq s\right\}$, where $C_{t}\left(a, x_{i}\right)$ is as in Lemma 4.4. Let $n$ be the number of circles containing $a$ and tangent to both $C$ and $C^{\prime}$. If $n=0$ then we are done. Suppose $n>0$, i.e. $C^{\prime}$ is tangent to at least one circle in $\mathcal{C}$. Let $k$ be the number of circles in $\mathcal{C}$ disjoint from $C^{\prime}$. Then there are $s-n-k$ circles in $\mathcal{C}$ each having two points in common with $C^{\prime}$. Let $A=\left\{\left(y, C_{t}\left(a, x_{i}\right)\right) \mid y \mathbf{I} C^{\prime}, y \mathbf{I} C_{t}\left(a, x_{i}\right), 1 \leq i \leq s\right\}$. We show that $n$ is even by counting $|A|$ in two ways. Thus let $m$ be the number of points on $C^{\prime}$ incident with a circle in $\mathcal{C}$. By Lemma 4.4 (i), each of the $\mathrm{m}$ points is on exactly two circles in $\mathcal{C}$. It follows that $2 m=n+2(s-n-k)$ and so $n$ is even.

(ii) (a) Let $\left\{x_{0}, x_{1}, \ldots, x_{s}\right\}$ and $\left\{x_{0}, x_{1}, y_{2}, \ldots, y_{s}\right\}$ be the sets of points on $C$ and $C^{\prime}$ respectively, where without loss of generality we may assume that $x_{0}$ is collinear with $a$. Define $n, k, m$ and $A$ as in the proof of part (i). Note that Lemma 4.4 (i) applies to the points of $C^{\prime}$ except $x_{0}$ and $x_{1}$ and that $x_{1} \mathbf{I} C_{t}\left(a, x_{1}\right)$. It follows that $2(m-1)+1=n+2(s-n-k)$ and so $n$ is odd.

(b) Let $\left\{x_{0}, x_{1}, \ldots, x_{s}\right\}$ and $\left\{x_{0}, x_{1}, y_{2}, \ldots, y_{s}\right\}$ be the sets of points on $C$ and $C^{\prime}$ respectively, where without loss of generality we may assume that $x_{2}$ and $y_{2}$ are collinear with $a$. Let $\mathcal{C}=\left\{C_{t}\left(a, x_{i}\right) \mid 0 \leq i \leq s, i \neq 2\right\}$. Define $n, k, m$ and $A$ as in the proof of part (i). In this case $2(m-2)+2=n+2(s-n-k)$ and so $n$ is even.

\section{A geometric proof of Theorem 3.3}

In this section we give a proof of Theorem 3.3 using Laguerre geometry. Thus, let $\mathcal{S}=(\mathcal{P}$, $\mathcal{B}, \mathbf{I})$ be a GQ of (odd) order $s$ with an antiregular point $z \in \mathcal{P}$. Let $z^{\prime}$ be another point. We want to prove that $z^{\prime}$ is antiregular.

First we consider the case where $z^{\prime} \in z^{\perp} \backslash\{z\}$. Let $u, u^{\prime} \in \mathcal{P} \backslash\left\{z^{\prime}\right\}$ be two distinct points such that $z^{\prime} \nsim u \nsim u^{\prime} \nsim z^{\prime}$. To prove that $z^{\prime}$ is antiregular, it suffices to show that $\left|z^{\prime \perp} \cap u^{\perp} \cap u^{\prime \perp}\right|$ is even. Indeed, from the usual counting argument presented in the proof of Theorem 2.7, this implies that $\left|z^{\prime \perp} \cap u^{\perp} \cap u^{\prime \perp}\right|=0$ or 2 and Lemma 2.3 applies. There are three cases to consider: (i) $\left|\left\{u, u^{\prime}\right\} \cap z^{\perp}\right|=2$, (ii) $\left|\left\{u, u^{\prime}\right\} \cap z^{\perp}\right|=1$, and (iii) $\left|\left\{u, u^{\prime}\right\} \cap z^{\perp}\right|=0$. For (i), as $z$ is antiregular, $\left|z^{\prime \perp} \cap u^{\perp} \cap u^{\prime \perp}\right|=2$. For (ii), we may assume without loss of generality that $u \in z^{\perp}$ and $u^{\prime} \notin z^{\perp}$. If $\left|z^{\prime \perp} \cap u^{\perp} \cap u^{\prime \perp}\right|=0$, then we are done. Suppose $\left|z^{\prime \perp} \cap u^{\perp} \cap u^{\prime \perp}\right| \geq 1$. Let $v \in z^{\prime \perp} \cap u^{\perp} \cap u^{\prime \perp}$. Applying Lemma 4.3 with $z^{\prime}, u, u^{\prime}$ and $v$ respectively playing the roles of $y, z, C^{\prime}$ and $C$ of Lemma 4.3, we obtain in the Laguerre plane $\mathcal{L}$ a unique circle $C^{\prime \prime}$ containing $y, z$ and tangent to $C^{\prime}$, i.e. in $\mathcal{S}$ a unique point, say $w$, which is in $z^{\perp} \cap u^{\perp} \cap u^{\prime \perp}$ and different from $v$. Thus $\left|z^{\prime \perp} \cap u^{\perp} \cap{u^{\prime}}^{\perp}\right|=2$. For (iii), by applying Lemma 4.5 with $z^{\prime}, u, u^{\prime}$ playing the roles of $a, C, C^{\prime}$ respectively, we conclude that $\left|z^{\prime \perp} \cap u^{\perp} \cap u^{\prime \perp}\right|$ is even.

The other case is where $z^{\prime} \in \mathcal{P} \backslash z^{\perp}$. Let $\operatorname{tr}\left(z, z^{\prime}\right)=\left\{u_{1}, \ldots, u_{s+1}\right\}$. Since $u_{1} \in z^{\perp} \backslash\{z\}$ and $z^{\prime} \in u_{1}^{\perp} \backslash\left\{u_{1}\right\}$, we are back in the previous case, and so the antiregularity of $u_{1}$ follows from that of $z$, and the antiregularity of $z^{\prime}$ follows from that of $u_{1}$. As a result, every point of $\mathcal{S}$ is antiregular.

This completes the geometric proof of Theorem 3.3.

Acknowledgment The authors would like to thank the reviewers for their suggestions which lead to a shortening of the proof of our main result.

Open Access This article is distributed under the terms of the Creative Commons Attribution Noncommercial License which permits any noncommercial use, distribution, and reproduction in any medium, provided the original author(s) and source are credited. 


\section{References}

1. Bagchi B., Brouwer A.E., Wilbrink H.A.: Notes on binary codes related to the $O(5, q)$ generalized quadrangle for odd $q$. Geom. Dedicata 39, 339-355 (1991).

2. Benson C.T.: On the structure of generalized quadrangles. J. Algebra 15, 443-454 (1970).

3. De Soete M., Thas J.A.: A characterization of the generalized quadrangle $Q(4, q), q$ odd. J. Geom. 28, 57-79 (1987).

4. Hirschfeld J.W.P., Thas J.A.: General Galois Geometries, Oxford Mathematical Monographs. Oxford Science Publications, The Clarendon Press, Oxford University Press, Oxford (1991).

5. Hughes D.R., Piper F.C.: Projective Planes. GTM 6. Springer, Berlin (1973).

6. Mazzocca F.: Sistemi grafici rigati di seconda specie. 1st Mat. Univ. Napoli Rel. 28 (1973).

7. Payne S.E., Thas J.A.: Generalized quadrangles with symmetry, Part II. Simon Stevin 49, 81-103 (1976).

8. Payne S.E., Thas J.A.: Finite Generalized Quadrangles, 2nd edn. European Mathematical Society (2009).

9. Thas J.A.: Circle Geometries and Generalized Quadrangles. Finite Geometries, pp. 327-352. Dekker, New York (1985).

10. Thas J.A., Thas K., Van Maldeghem H.: Translation Generalized Quadrangles. Ser. Pure Math. 26, World Scientific Publishing Co. Pte. Ltd., London WC2H 9HE (2006). 